\title{
SELETIVIDADE DA REDE DE ESPERA UTILIZADA NA CAPTURA DA SARDINHA-BANDEIRA, Opisthonema oglinum (LESUEUR, 1818)
}

\author{
Selectivity of the gill net used in the capture of the atlantic \\ thread-herring, Opisthonema oglinum (Lesueur, 1818)
}

\author{
Sebastião Ribeiro D’Alva Teixeira1*, Reynaldo Amorim Marinho², \\ Raimundo Nonato de Lima Conceição ${ }^{3}$, Silvana Saker-Sampaio ${ }^{4}$ \\ ${ }^{1}$ Doutorando em Engenharia de Pesca, Universidade Federal do Ceará, bolsista Demanda Social da Capes. \\ E-mail: seridaltex@hotmail.com \\ ${ }^{2}$ Professor associado I, Departamento de Engenharia, Universidade Federal do Ceará. \\ E-mail: marinho.rey@gmail.com \\ ${ }^{3}$ Professor associado III, Departamento de Engenharia de Pesca, Universidade Federal do Ceará. \\ E-mail: nonatodelima@ufc.br \\ ${ }^{4}$ Professor titular, Departamento de Engenharia de Pesca, Universidade Federal do Ceará. \\ E-mail: sakersil@gmail.com - *autor para correspondência
}

\begin{abstract}
RESUMO
O presente trabalho teve como objetivo determinar a seletividade das redes de espera utilizadas na captura da sardinha-bandeira, Opisthonema oglinum, na Praia da Caponga, município de Cascavel, Ceará, Brasil. Os dados de comprimento total $(\mathrm{em} \mathrm{cm})$ foram obtidos de dois grupos, cada um com 780 indivíduos, capturados por redes de espera com malhas de $5 \mathrm{~cm}$ e $6 \mathrm{~cm}$ entre nós opostos, no período de agosto a outubro de 2014. Esses dados foram descritos em histogramas de frequência de comprimento, sendo mais representativas as classes de $21,6 \mathrm{~cm}(24,23 \%)$ para a malha de $5 \mathrm{~cm}$ e de $25,7 \mathrm{~cm}(23,08 \%)$ para a de $6 \mathrm{~cm}$. O comprimento médio de seleção foi de $23,7 \mathrm{~cm}$ para a malha de $5 \mathrm{~cm}$ e de 28,5 $\mathrm{cm}$ para a de $6 \mathrm{~cm}$. A amplitude de seleção foi de $23,7 \pm 7,9 \mathrm{~cm}$ e de $28,5 \pm 7,9 \mathrm{~cm}$ para as malhas de $5 \mathrm{~cm}$ e $6 \mathrm{~cm}$, respectivamente. As equações das curvas de seleção das redes de espera para os dois tamanhos de malha foram obtidas, e a probabilidade de capturar indivíduos com comprimento inferior ao mínimo, estabelecido pela Instrução Normativa no 53 , de $22 / 11 / 2005$, em $15 \mathrm{~cm}$, foi muito baixa e correspondeu a 1,32\% e 0,03\% para as malhas de $5 \mathrm{~cm}$ e de $6 \mathrm{~cm}$, respectivamente.
\end{abstract}

Palavras-chave: sardinha-de-lage, biologia pesqueira, estrutura da população, curva de seleção, rede de espera.

Recebido em: 19/02/2019 


\section{ABSTRACT}

The present research aims to determine the selectivity of the gill nets used in capture of the atlantic thread-herring, Opisthonema oglinum, from Caponga beach, municipality of Cascavel, Ceará, Brazil. Data of total length $(\mathrm{cm})$ were obtained from two groups, each with 780 individuals, captured by gill nets with $5 \mathrm{~cm}$ and $6 \mathrm{~cm}$ meshes between opposing nodes, from August to October 2014. These data were described in histograms of frequency of length, being more representatives the classes of $21.6 \mathrm{~cm}(24.23 \%)$ for the mesh of $5 \mathrm{~cm}$ and of $25.7 \mathrm{~cm}(23.08 \%)$ for the one of $6 \mathrm{~cm}$. The mean selection length was $23.7 \mathrm{~cm}$ for the $5 \mathrm{~cm}$ mesh and $28.5 \mathrm{~cm}$ for the $6 \mathrm{~cm}$ mesh. The selection range was $23.7 \pm 7.9 \mathrm{~cm}$ and $28.5 \pm 7.9 \mathrm{~cm}$ for the $5 \mathrm{~cm}$ and $6 \mathrm{~cm}$ mesh, respectively. The equations of the grid selection curves for the two mesh sizes were obtained, and the probability of capturing individuals with less than the minimum length established in $15 \mathrm{~cm}$ was very low and corresponded to $1.32 \%$ and $0.3 \%$ for $5 \mathrm{~cm}$ and $6 \mathrm{~cm}$ mesh, respectively.

Keywords: atlantic thread-herring, fisheries biology, population structure, selection curve, gill nets.

\section{INTRODUÇÃO}

A sardinha-bandeira, Opisthonema oglinum (Lesueur, 1818), é uma das cinco espécies do gênero Opisthonema que pertence à família Clupeidae da ordem Clupeiformes. Conhecida por uma enorme variedade de nomes vulgares (sardinha, sardinha-de-laje, manjuba, sargo, xangó e muitos outros), é um teleósteo marinho de pequeno porte, cuja ocorrência está associada a recifes (Froese \& Pauly, 2009).

A distribuição geográfica da sardinha-bandeira está registrada na costa leste das Américas do Norte, Central eSul, desde o Golfo do Maine, nos Estados Unidos, e Bermudas, até o Golfo do México, Caribe, as Índias ocidentais e o Brasil (Whitehead, 1985).

No Brasil, até o final do século XIX, a pesca da sardinha não tinha importância econômica, sendo realizada por pescadores artesanais apenas com a finalidade de alimentar as populações costeiras. No entanto, nas primeiras décadas do século $X X$, esse quadro se modificou, e a atividade pesqueira assumiu um caráter de pesca de grande escala com valor comercial. A captura de sardinhas nas regiões Sul e Sudeste é feita em escala industrial pela frota de traineiras, tendo como espécie-alvo a sardinha-verdadeira, Sardinella brasiliensis; enquanto no Nordeste brasileiro, a pesca da sardinha ainda é predominantemente artesanal, tendo como espécie-alvo a sardinha-bandeira, O. oglinum (Vieira et al., 2010).

Considerada uma fonte de alimento abundante e de baixo custo (Figueiredo \& Menezes, 1978; Lino, 2003), o valor nutricional da sardinha é reconhecido não só pela elevada quantidade de ácidos graxos poli-insaturados e pelo baixo teor de colesterol mas também por ser fonte de proteína de alto valor biológico, possuindo também quantidades significativas de vitaminas A, B6, B12 e niacina e de minerais como cálcio, ferro, fósforo, magnésio e sódio (Capistrano-Sobrinho et al., 2011).

São habitantes de áreas costeiras rasas e formam cardumes. Embora tenha hábito pelágico costeiro, na época da desova, a sardinha-bandeira costuma se aproximar do litoral para, em seguida, se deslocar para o mar aberto, comportamento comum aos clupeídeos (Ihering, 1932). De acordo com Menezes (1968), a sardinha-bandeira pode ser capturada durante todo o ano na costa cearense, mas as melhores pescarias são realizadas entre os 
meses de maio e dezembro. No estudo de Mota-Alves e Sawaya (1975), o aspecto histológico dos ovários indica que a sardinha-bandeira apresenta desova total nas águas litorâneas do estado do Ceará, ocorrendo uma vez por ano, de junho a novembro, com maior intensidade em agosto e setembro.

De acordo com o Boletim Estatístico da Pesca e Aquicultura (Brasil, 2013), a produção nacional de sardinha foi 9.237,2 t em 2009, 8.709,5 t em 2010 e 8.810,3 t em 2011, sem especificação por unidade federativa. O levantamento mais recente da produção de pescado marítimo e estuarino do estado do Ceará refere-se ao ano de 2007 (17.920 t). Dessa produção, a sardinha-bandeira contribuiu com 1.270,5 t, ou seja, cerca de 7\% do total (Brasil, 2007).

Na Praia da Caponga, município de Cascavel, no Ceará, uma das principais atividades econômicas desenvolvidas pela população é a pesca marítima artesanal, que é exercida como pesca comercial de pequena escala para prover a subsistência de muitas famílias (Castro \& Silva, 2004; Kobayashi, 2000). Nessa praia, a pesca da sardinha-bandeira é realizada principalmente com redes de espera e, em uma escala quase insignificante, com linha de mão. As embarcações utilizadas na captura da sardinha-bandeira nessa localidade são denominadas paquetes, todas movidas a vela, com comprimento variando entre $2,5 \mathrm{~m}$ e 5 m. A pescaria é realizada de 5 a 6 dias por semana, sendo que, para cada expedição pesqueira, viajam, na maioria das vezes, dois pescadores por embarcação (Sá, 2010). Não obstante, a Praia da Caponga figura entre os oito principais portos de desembarque de pescado no Ceará (Brasil, 2006).

No Brasil, estudos sobre a seletividade de rede de espera utilizada na capturada da sardinha-bandeira são ainda incipientes, apesar da importância dessa espécie na pesca regional. A falta de legislação para a pesca da sardinha-bandeira com redes de espera deve-se em parte à incipiência de estudos sobre o funcionamento e a eficiência das artes utilizadas. Entre poucos trabalhos sobre a seletividade da rede de espera utilizada na captura da sardinha-bandeira no Brasil destacam-se os de Teixeira (2013) e Lima e Andrade (2018). Teixeira (2013) estudou a estrutura da população da sardinha-bandeira e a seletividade das redes de espera de malhas de $5 \mathrm{~cm}$ e $5,5 \mathrm{~cm}$, utlizadas na sua captura no município de Cascavel. Lima e Andrade (2018) determinaram a seletividade das redes de espera de malhas de $3 \mathrm{~cm}, 4 \mathrm{~cm}$ e $5 \mathrm{~cm}$, utilizadas para capturar peixes forrageiros, com ênfase para Opisthonema oglinum no complexo estuarino do Canal de Santa Cruz, em Pernambuco.

De acordo com Fonteles-Filho (2011), raramente a pesca explora toda a população de uma espécie, mas apenas aqueles indivíduos que estão dentro de determinadas faixas de comprimento e idade, o estoque disponível. Deste, a pesca explora somente aquela porção que está acessível ao aparelho de pesca, o estoque capturável. O ideal seria que os aparelhos de pesca capturassem apenas os indivíduos já sexualmente maduros, mas, na prática, o estoque capturável é constituído do estoque adulto mais uma parte do estoque jovem, sendo que a participação relativa deste na captura é determinada pelas características seletivas dos aparelhos de pesca.

Para uma gestão pesqueira sustentável são necessárias informações básicas, as quais são fornecidas pela avaliação dos estoques, levando-se em consideração os processos de dinâmica populacional capazes de afetar a biomassa das populações (Castello, 2007). O ordenamento pesqueiro inclui diferentes medidas, entre elas o controle e a regulamentação sobre as artes de pesca, que poderão alterar sua seletividade (Bjordal, 2005).

Nesse contexto, o objetivo do presente trabalho consistiu em determinar a seletividade das redes de espera utilizadas na captura da sardinha-bandeira, Opisthonema oglinum 
(Lesueur, 1818), na Praia da Caponga, destacando o comprimento total, o comprimento médio de seleção, a amplitude da seleção e a curva de seleção.

\section{MATERIAL E MÉTODOS}

\section{Caracterização da área de estudo}

A Praia da Caponga está localizada no município de Cascavel, distrito de Caponga, no litoral leste do estado do Ceará, distante $61 \mathrm{~km}$ da capital Fortaleza (Figura 1). O município de Cascavel limita-se ao norte com o Oceano Atlântico, ao sul com Ocara, a leste com Beberibe e a oeste com Horizonte, Pacajús e Chorozinho. Possui clima tropical quente semiárido brando com pluviosidade de $1.331,7 \mathrm{~mm}$ no período de janeiro a maio e temperatura média oscilando entre $26^{\circ} \mathrm{C}$ e $28^{\circ} \mathrm{C}$ (Ceará, 2013).

Caponga é o distrito de maior adensamento populacional no litoral cascavelense, com $60 \%$ da ocupação, compreendendo as praias Águas Belas, Balbino e Caponga (Castro \& Silva, 2004). A economia desse distrito é baseada principalmente na produção de pescado, mas, nos últimos anos, o turismo tem contribuído de forma importante como alternativa de emprego e renda (Ceará, 2013).

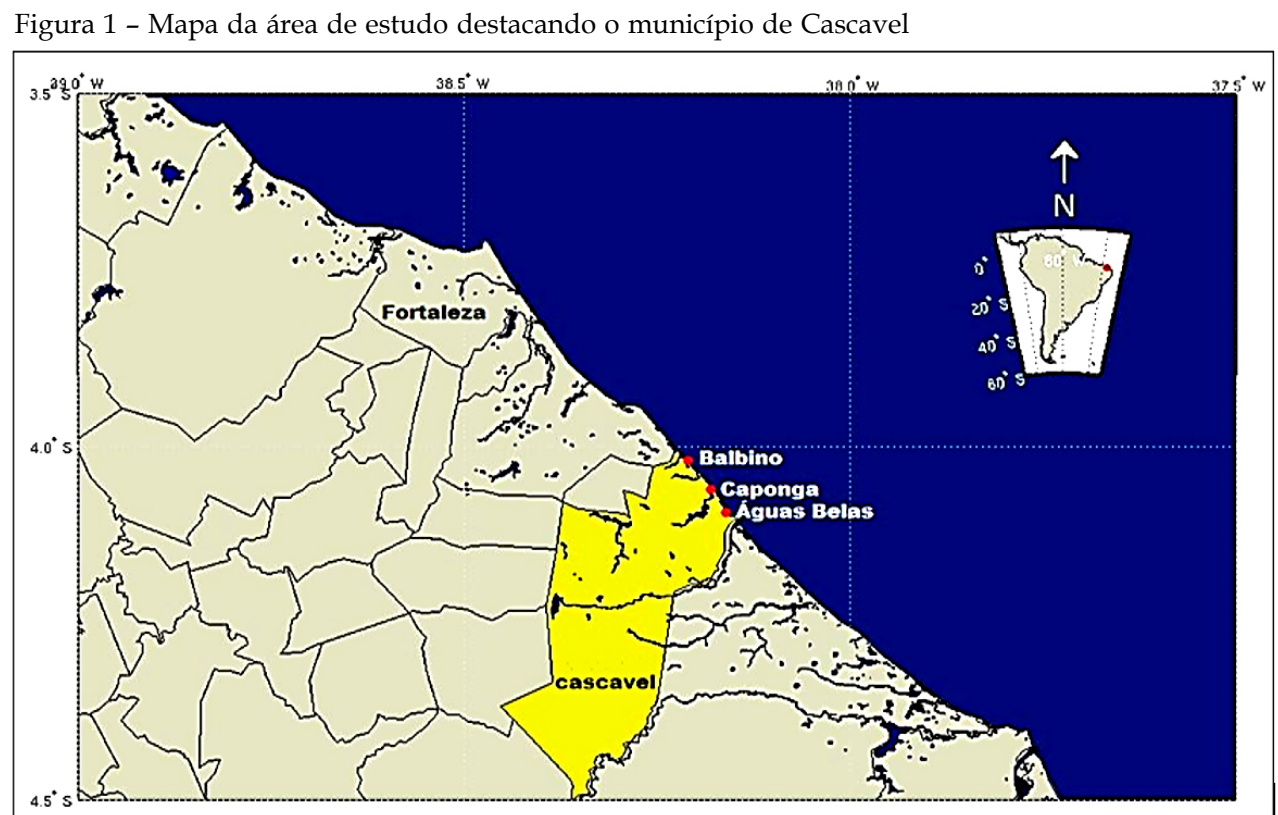

Fonte: Sá (2010).

\section{Amostragem e coleta de dados}

Para a identificação da espécie, cinco exemplares de sardinha-bandeira foram adquiridos na Praia da Caponga, sendo resfriados, acondicionados em caixa térmica e transportados para o Laboratório de Biologia Pesqueira do Departamento de Engenharia de Pesca da Universidade Federal do Ceará, onde foram identificados de acordo com o Manual de Peixes Marinhos do Sudeste do Brasil (Figueiredo \& Menezes, 1978), o Guia de Identificação de Peixes Marinhos da Região Nordeste do Brasil (Lessa \& Nóbrega, 2000) e o FishBase (2019).

Os indivíduos foram capturados no período de agosto a outubro de 2014 pela embarcação comercial (paquete) Jamilson II, movida a vela, com comprimento total de 3,6 m e largura da boca moldada de 1,75 m. A coleta dos dados foi procedida durante o desem- 
barque dessa embarcação. Cada expedição pesqueira, com duração média de 5 horas, era realizada diariamente, com exceção de domingo, por dois pescadores a bordo.

A embarcação operava simultaneamente com quatro redes de espera, acopladas umas às outras, cada uma medindo $50 \mathrm{~m}$ de comprimento e $2 \mathrm{~m}$ de altura, contendo 50 flutuadores de isopor na tralha superior distantes $1 \mathrm{~m}$ entre eles e aproximadamente $3 \mathrm{~kg}$ de chumbada distribuídos ao longo da tralha inferior.

As redes, fabricadas de monofilamento de poliamida de $0,30 \mathrm{~mm}$ de diâmetro, tinham malha de dois tamanhos diferentes: duas com malha de $5 \mathrm{~cm}$ entre nós opostos e as outras duas com malha de $6 \mathrm{~cm}$ entre nós opostos.

Cada coleta quinzenal consistiu em uma amostra aleatória de 130 indivíduos, totalizando 780 exemplares com a rede de $5 \mathrm{~cm}$ de malha e $780 \mathrm{com}$ a de $6 \mathrm{~cm}$ de malha. Os dados de comprimento total foram obtidos com o auxílio de um ictiômetro, com precisão de 1 milímetro.

\section{Análise dos dados}

Para o estudo da estrutura da população, primeiramente os conjuntos de dados de comprimento total foram caracterizados de acordo com a Estatística Descritiva, em tabelas de distribuição de frequência e histogramas de frequência, separadamente para cada tamanho de malha (780 indivíduos para a de $5 \mathrm{~cm}$ e 780 indivíduos para a de $6 \mathrm{~cm}$ ).

Em seguida, os dados de comprimento total foram reunidos sem levar em consideração o tamanho de malha (1.560 indivíduos) e, igualmente, descritos de acordo com o que preconiza a Estatística Descritiva.

As médias de comprimento total obtidas para cada tamanho de malha foram comparadas pelo teste $t$ de Student para dados independentes, utilizando o programa gratuito BioEstat na versão 5.3 (Instituto Mamirauá), atendidos todos os pressupostos de normalidade e homocedasticidade.

O estudo da seletividade das redes de espera utilizadas na captura da sardinha-bandeira na Praia da Caponga foi determinado pelo modelo de Holt (1963). Para isso, os comprimentos médios de seleção $(l c)$, as amplitudes da seleção $(A m)$ e as curvas de seleção foram determinados para os dois tamanho de malha $\left(m_{5}=5 \mathrm{~cm}\right.$ e $\left.m_{6}=6 \mathrm{~cm}\right)$.

\section{Comprimento médio de seleção}

Os comprimentos médios de seleção $(l c)$ para cada tamanho de malha foram calculados pela Equação 1, em que a constante $K$ (Equação 2) foi multiplicada pelo tamanho da malha $(m)$.

$$
\begin{gathered}
l c=K x m \\
\mathrm{~K}=\frac{-2 a}{b\left(m_{6}+m_{5}\right)}
\end{gathered}
$$

Em que:

$a=$ coeficiente linear

$b=$ coeficiente angular

$m_{6}=$ tamanho da malha maior $(6 \mathrm{~cm})$

$m_{5}=$ tamanho da malha menor $(5 \mathrm{~cm})$ 


\section{Amplitude da seleção}

As amplitudes da seleção $(A m)$ foram determinadas pela Equação 3, considerando-se o intervalo delimitado por 1,96 desvios padrão em torno do comprimento médio de seleção (lc), para cada tamanho de malha, dentro do qual 95\% dos indivíduos são capturáveis, admitindo-se curvas de seleção unimodais.

O desvio padrão das curvas de seleção (s) foi obtido tirando-se a raiz quadrada de sua variância $\left(s^{2}\right)$, calculada pela Equação 4 .

$$
\begin{aligned}
& A m=l c \pm 1,96 s \\
& s^{2}=\frac{-2 a\left(m_{6}-m_{5}\right)}{b^{2}\left(m_{6}-m_{5}\right)}
\end{aligned}
$$

Curvas de seleção

As curvas de seleção das redes de espera foram determinadas de acordo com Holt (1963) e podem ser representadas pela Equação 5, simplificada da função-densidade da distribuição normal. Para a determinação dessas curvas foram considerados todos os indivíduos (1.560) capturados nas redes de espera de $5 \mathrm{~cm}$ e $6 \mathrm{~cm}$ de malha entre nós opostos.

$$
P(l)=e^{-\frac{(l-l c)^{2}}{2 s^{2}}}
$$

\footnotetext{
Em que:

$P(l)=$ probabilidade de captura de indivíduos com comprimento $l$

$e=$ base dos logaritmos neperianos

$l=$ um valor de comprimento

$l c=$ comprimento médio de seleção

$s^{2}=$ variância da curva de seleção
}

A partir dos dados de comprimento total estabeleceu-se uma regressão linear entre este (classe de comprimento total dos indivíduos) e o logaritmo neperiano da razão entre o número de indivíduos capturados nas redes de malha de $6 \mathrm{~cm}$ e $5 \mathrm{~cm}$, permitindo, assim, a determinação do coeficiente angular $(b)$, do coeficiente linear $(a)$ e do coeficiente de correlação de Pearson $(r)$.

Para determinar a probabilidade de as redes de espera de malhas de $5 \mathrm{~cm}$ e $6 \mathrm{~cm}$ entre nós opostos capturarem indivíduos com comprimento abaixo do comprimento mínimo de captura (lmc), foi aplicado o teste $z$ (Equação 6).

$$
Z=\frac{l m c-l c}{s}
$$




\section{RESULTADOS E DISCUSSÃO}

A sardinha-bandeira, Opisthonema oglinum, apresentou corpo prateado lateralmente e sem linha lateral; mancha negra arredondada na parte superior da margem da câmara branquial acompanhada de outras relativamente menores e alinhadas horizontalmente; número de raios nadadeira anal (sem espinhos) entre 21 e 26; e número de raios nadadeira dorsal (sem espinhos) entre 19 e 21, sendo o último filamentoso e prolongado (Figueiredo \& Menezes, 1978; Lessa \& Nóbrega, 2000; Froese \& Pauly, 2019) (Figura 2).

Figura 2-Exemplar desardinha-bandeira, Opisthonema oglinum (Lesueur, 1818), capturada na Praia da Caponga

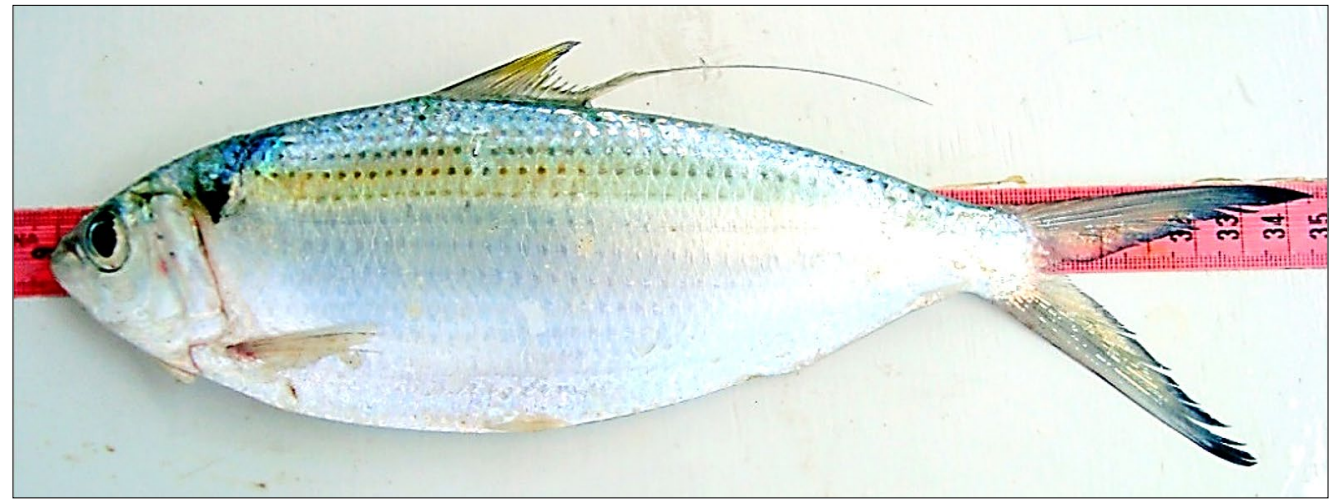

Os dados de comprimento total dos indivíduos capturados com rede de espera de malha de $5 \mathrm{~cm}$ e $6 \mathrm{~cm}$ entre nós opostos foram agrupados em tabelas de distribuição de frequência com 11 classes e amplitude igual a 1,4 cm. Os comprimentos totais médios dos 780 indivíduos capturados com a malha de $5 \mathrm{~cm}$ e $6 \mathrm{~cm}$ foram, respectivamente, $23,4 \mathrm{~cm} \pm 2,4$ $\mathrm{cm}$ e $25,9 \mathrm{~cm} \pm 2,3 \mathrm{~cm}$. Os centros de classe para a malha de $5 \mathrm{~cm}$ variaram de $18,8 \mathrm{~cm}$ a 32,8 $\mathrm{cm}$, sendo mais representativas as classes cujos valores médios foram: 21,6 cm com 189 indivíduos (24,23\%), 23 cm com 186 indivíduos (23,85\%) e 24,4 cm com 122 indivíduos (15,64\%) (Figura 3). Para a malha de $6 \mathrm{~cm}$, esses valores variaram de $20,1 \mathrm{~cm}$ a $34,1 \mathrm{~cm}$, sendo mais representativas as classes cujos valores médios foram: $24,3 \mathrm{~cm}$ com 154 indivíduos (19,74\%), 25,7 cm com 180 indivíduos (23,08\%) e 27,1 cm com 149 indivíduos (19,10\%) (Figura 4).

Figura 3 - Distribuição de frequência dos dados de comprimento total da sardinha-bandeira, Opisthonema oglinum (Lesueur, 1818), capturada com rede de espera de malha de $5 \mathrm{~cm}$ entre nós opostos, na Praia da Caponga, de agosto a outubro de 2014

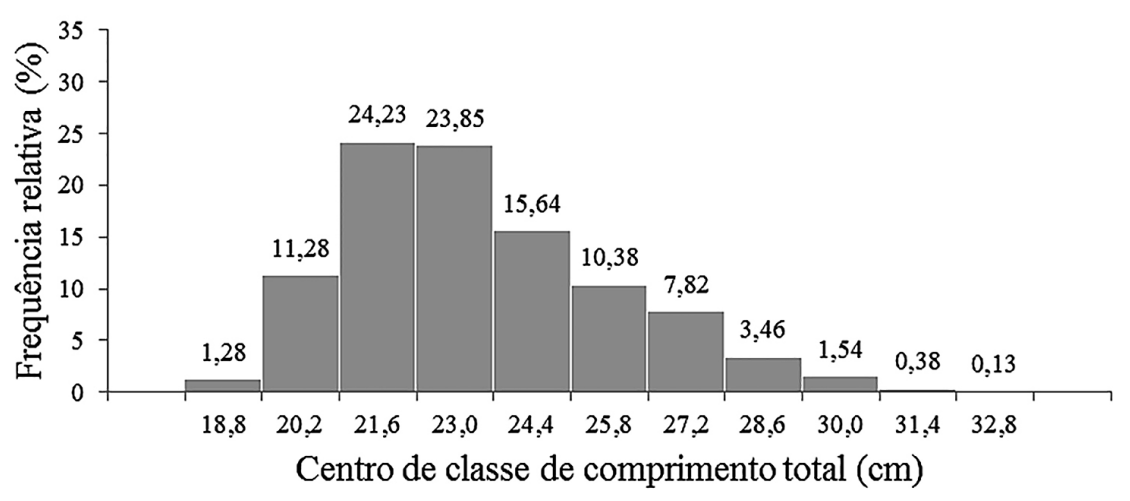


Figura 4 - Distribuição de frequência dos dados de comprimento total da sardinha-bandeira, Opisthonema oglinum (Lesueur, 1818), capturada com rede de espera de malha de $6 \mathrm{~cm}$ entre nós opostos, na Praia da Caponga, de agosto a outubro de 2014

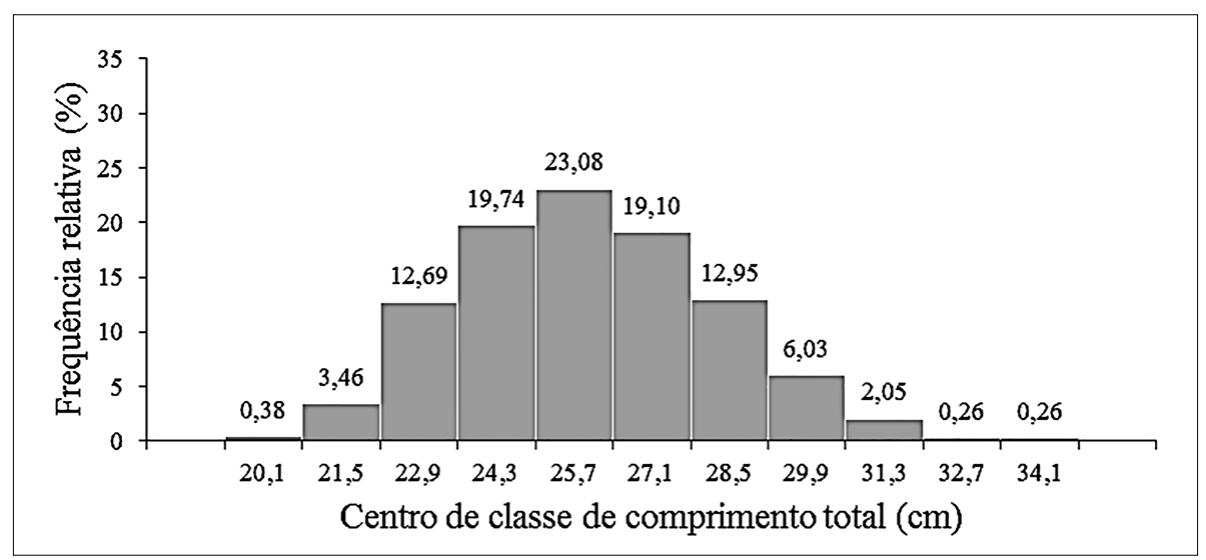

O teste $t$ de Student para dados independentes revelou que o comprimento total médio dos indivíduos capturados com a rede de malha maior foi estatisticamente superior ao dos indivíduos capturados com a rede de malha menor $(p<0,0001 ; t=-20,36)$. Esse foi um resultado esperado, considerando-se que os peixes maiores tendem a ser capturados por malhas maiores, salvo quando o peixe for capturado por emaranhamento, que é quando o peixe se prende por alguma parte do corpo, sem necessariamente ter penetrado na malha (Martins et al., 2011).

Os resultados do estudo biométrico (comprimento total) estão apresentados na Tabela I, com média, desvio padrão, valores máximos, valores mínimos e amplitude total de cada variável estudada.

Tabela I - Estatística descritiva para a variável morfométrica obtida para a sardinha-bandeira, Opisthonema oglinum (Lesueur, 1818), capturada com redes de espera (malhas de $5 \mathrm{~cm}$ e $6 \mathrm{~cm}$ entre nós opostos e agrupadas), na Praia da Caponga, de agosto a outubro de 2014

\begin{tabular}{c|c|c|c|c|c}
\hline \multirow{2}{*}{ Tamanho da malha } & \multicolumn{5}{|c}{ Comprimento total $(\mathrm{cm})$} \\
\cline { 2 - 7 } & Média & $\begin{array}{c}\text { Desvio } \\
\text { padrão }\end{array}$ & $\begin{array}{c}\text { Valor } \\
\text { mínimo }\end{array}$ & $\begin{array}{c}\text { Valor } \\
\text { máximo }\end{array}$ & $\begin{array}{c}\text { Amplitude } \\
\text { total }\end{array}$ \\
\hline $5 \mathrm{~cm}$ & 23,4 & 2,4 & 18,8 & 32,8 & 14 \\
\hline $6 \mathrm{~cm}$ & 25,9 & 2,3 & 20,1 & 34,0 & 13,9 \\
\hline $5 \mathrm{~cm} \mathrm{e} 6 \mathrm{~cm}$ juntas & 24,7 & 2,7 & 18,8 & 34,0 & 15,2 \\
\hline
\end{tabular}

Fonte: elaborada pelo autor.

Os dados de comprimento total dos 1.560 indivíduos capturados com as rede de espera (malha de $5 \mathrm{~cm}$ e $6 \mathrm{~cm}$ entre nós opostos) foram agrupados em uma tabela de distribuição de frequência com 12 classes, com amplitude de 1,4 cm. Os centros de classe variaram de $18,8 \mathrm{~cm}$ a $34,2 \mathrm{~cm}$, sendo mais representativas as classes cujos valores médios foram de $23 \mathrm{~cm}, 24,4 \mathrm{~cm}$ e 25,8 cm, com respectivamente 290 indivíduos (18,59\%), 285 indivíduos $(18,27 \%)$ e 256 indivíduos (16,41\%), como mostrado na Figura 5.

Neste trabalho, o comprimento total máximo encontrado para a sardinha-bandeira durante o período estudado foi de $34 \mathrm{~cm}$ (Tabela I), inferior ao citado no Froese e Pauly (2019), de 38 cm, na costa norte da América do Sul, entre a Colômbia e a Guiana Francesa. 
Entretanto, esse valor foi maior do que os referidos para a mesma espécie na região Nordeste, de $22 \mathrm{~cm}$ (Lino, 2003) e de 28,5 cm (Teixeira, 2013), assim como o da região Sudeste, que correspondeu a $30 \mathrm{~cm}$ (Figueiredo \& Menezes, 1978). Isso mostra que o comprimento total máximo da sardinha-bandeira, como era de se esperar, varia de uma região para outra ou dentro de uma mesma região, dependendo da época do ano, da área de captura e das características de cada rede de espera.

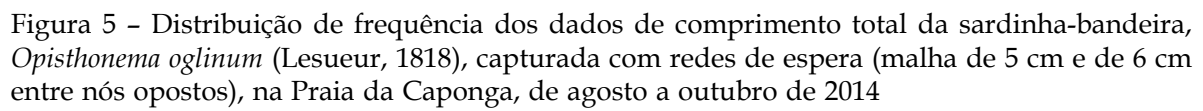
entre nós opostos), na Praia da Caponga, de agosto a outubro de 2014

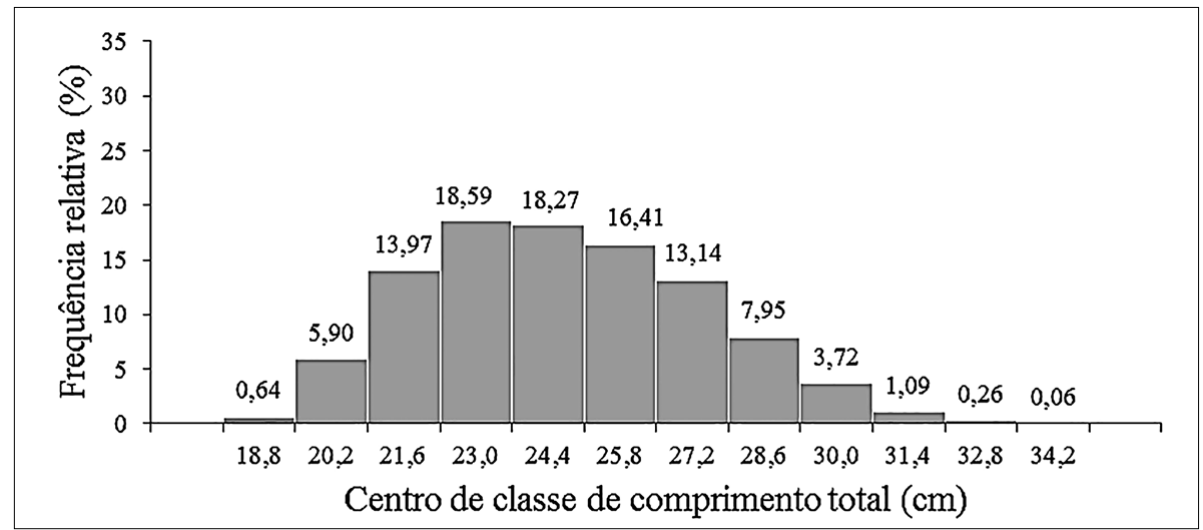

Estabeleceu-se uma regressão linear entre o comprimento total dos indivíduos, representado pelos centros de classe (variável $x$ ), e o logaritmo neperiano da razão entre o número de indivíduos capturados com a malha maior $(6 \mathrm{~cm})$ e com a menor $(5 \mathrm{~cm})$ (variável y). Para melhor compreensão, ver a Tabela II. A partir dos dados apresentados na Tabela II, calcularam-se os coeficientes da equação de regressão linear, obtendo-se os seguintes resultados para os coeficientes linear $(a=-7,981)$, angular $(b=0,306)$ e de correlação de Pearson $(r=0,8493)\left[r_{\text {tabelado }}=0,602\right]$.

Tabela II - Frequências absolutas e logaritmos da razão entre as frequências para os diferentes tamanhos da malha, por centro de classe de comprimento total, das redes de espera $(5 \mathrm{~cm}$ e $6 \mathrm{~cm}$ entre nós opostos), utilizadas na captura da sardinha-bandeira, Opisthonema oglinum (Lesueur, 1818), na Praia da Caponga, de agosto a outubro de 2014

\begin{tabular}{c|c|c|c}
\hline \multirow{2}{*}{$\begin{array}{c}\text { Centros de classe de } \\
\text { comprimento total }(\mathrm{cm})(\boldsymbol{x})\end{array}$} & \multicolumn{2}{|c|}{ Número de indivíduos capturados } & \multirow{2}{*}{$\ln \left(\frac{m_{6}}{m_{5}}\right)(y)$} \\
\cline { 2 - 3 } & Malha de $5 \mathrm{~cm}\left(m_{5}\right)$ & Malha de $6 \mathrm{~cm}\left(m_{6}\right)$ & - \\
\hline 18,8 & 10 & - & $-3,091042453$ \\
20,2 & 88 & 4 & $-1,874451185$ \\
21,6 & 189 & 29 & $-0,581355775$ \\
23,0 & 186 & 104 & 0,289729156 \\
24,4 & 122 & 163 & 0,770336819 \\
25,8 & 81 & 175 & 0,858939435 \\
27,2 & 61 & 144 & 1,278874112 \\
28,6 & 27 & 97 & 1,343734747 \\
30,0 & 12 & 46 & 1,540445041 \\
31,4 & 3 & 14 & 0,693147181 \\
32,8 & 1 & 2 & - \\
34,2 & - & 2 & \\
\hline Total & 780 & 780 & \\
\hline
\end{tabular}

Fonte: elaborada pelo autor. 
O comprimento médio de seleção $(l c)$ foi, respectivamente, $23,7 \mathrm{~cm}$ e $28,5 \mathrm{~cm}$ para as redes de espera com malha de $5 \mathrm{~cm}$ e $6 \mathrm{~cm}$ entre nós opostos.

Considerando o desvio padrão igual a 3,939 cm, é possível afirmar com 95\% de confiança que as redes de espera com malhas de $5 \mathrm{~cm}$ e de $6 \mathrm{~cm}$ entre nós opostos capturaram indivíduos com comprimento total dentro do intervalo de $23,7 \pm 7,7 \mathrm{~cm}(16 \mathrm{~cm}$ a 31,4 cm, $A m=15,4 \mathrm{~cm})$ e de $28,5 \pm 7,7 \mathrm{~cm}(20,8 \mathrm{~cm}$ a $36,2 \mathrm{~cm}, A m=15,4 \mathrm{~cm})$, respectivamente.

A rede de espera, diferentemente das outras artes de pesca, possui dois comprimentos de seleção: um mínimo e um máximo. O primeiro seria aquele em que os peixes entram no intervalo de seleção e o segundo, o comprimento no qual o peixe cresce e abandona o intervalo de seleção (Puzzi \& Silva, 1981).

Camara et al. (1991) explicam que as capturas efetuadas pela rede em função do tamanho das malhas têm uma retenção máxima para certos comprimentos dos indivíduos (lc), caindo o seu poder de captura acima ou abaixo de determinados comprimentos. Esse intervalo representa a amplitude de seleção.

Substituindo-se os valores de comprimento médio de seleção $(l c)$ e de variância $\left(s^{2}\right)=$ $15,516 \mathrm{~cm}^{2}$, obtiveram-se as Equações 7 e 8, que permitiram os cálculos das probabilidades de captura que representam graficamente as curvas de seleção (Figura 6).

$$
\begin{aligned}
& P(l)=e^{-\frac{(l-23,7)^{2}}{31,032}} \quad \text { para malha de } 5 \mathrm{~cm} \\
& P(l)=e^{-\frac{(l-28,5)^{2}}{31,032}} \quad \text { para malha de } 6 \mathrm{~cm}
\end{aligned}
$$

Figura 6 - Curvas de seleção das redes de espera de malha de $5 \mathrm{~cm}$ e de $6 \mathrm{~cm}$ entre nós opostos utilizadas na captura da sardinha-bandeira, Opisthonema oglinum (Lesueur, 1818), na Praia da Caponga, de agosto a outubro de 2014

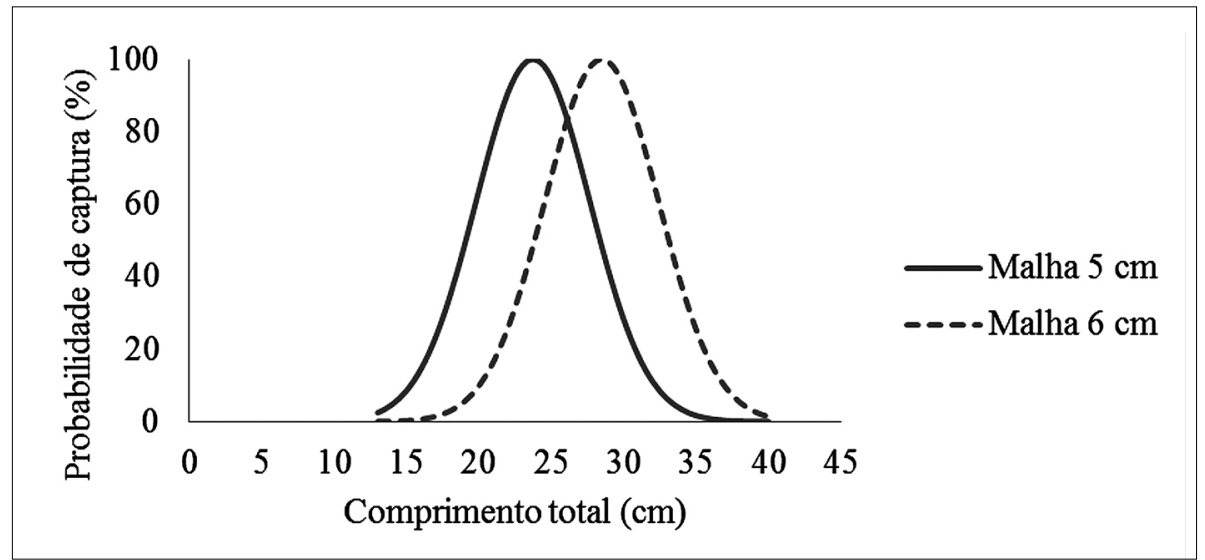

Todos os pressupostos do modelo de Holt (1963) do estudo da seletividade de redes de espera foram observados no presente trabalho: (1) curvas de seletividade com distribuição normal; (2) homocedasticidade; (3) tamanho ótimo de captura proporcional ao tamanho da malha; e (4) existência de sobreposição das curvas.

As curvas de seleção das redes de espera com as malhas de $5 \mathrm{~cm}$ e de $6 \mathrm{~cm}$ entre nós opostos alcançaram pontos máximos de probabilidade de captura nos comprimentos totais de $23,5 \mathrm{~cm}$ e $28,5 \mathrm{~cm}$, respectivamente, tendendo a zero à medida que os valores se aproximavam dos extremos da curva. 
Similarmente, as curvas de seleção obtidas para redes de espera de malha de $5 \mathrm{~cm}$ e de 5,5 cm utilizadas na captura da mesma espécie na Praia da Caponga revelaram pontos máximos de probabilidade de captura nos centros de classes de comprimento total de 21,9 $\mathrm{cm}$ e $23 \mathrm{~cm}$, respectivamente, tendendo a zero à medida que os valores se aproximaram dos extremos da curva (Teixeira, 2013).

Lima e Andrade (2018) obtiveram as curvas de seleção para redes de espera de malha de $3 \mathrm{~cm}, 4 \mathrm{~cm}$ e $5 \mathrm{~cm}$ utilizadas na captura da mesma espécie no complexo estuarino do Canal de Santa Cruz, em Pernambuco, com pontos máximos de probabilidade de captura nos comprimentos padrões de 10,31 cm, 13,76 cm e 17,50 cm, respectivamente, tendendo a zero à medida que os valores se aproximaram dos extremos da curva. Com base nesses dados, e utilizando a equação disponível no FishBase que estabelece a relação entre o comprimento padrão $(S L)$ e o comprimento total $(T L): S L=0+0,775 \times T L$, pode-se considerar que pontos máximos de probabilidade de captura da sardinha-bandeira determinados por Lima e Andrade (2018) foram nos comprimentos totais de 13,3 cm, 17,76 cm e 22,58 cm, respectivamente para as malhas de $3 \mathrm{~cm}, 4 \mathrm{~cm}$ e $5 \mathrm{~cm}$.

Comparando a curva de seleção da rede de espera de malha de $5 \mathrm{~cm}$ do presente trabalho com a do Lima e Andrade (2018), verificou-se que o ponto máximo de probabilidade de captura foi superior neste trabalho $(23,5 \mathrm{~cm}$ de comprimento total $)$ do que no trabalho de Lima e Andrade (2018) $(22,58 \mathrm{~cm})$.

A curva de seleção da rede de espera tem a forma típica da curva normal, ou seja, campaniforme, com um valor central máximo em torno do qual as frequências vão tendendo à zero. Isso significa que a probabilidade de captura é pequena nos extremos da distribuição normal, tanto para os indivíduos de pequeno porte como para os de grande porte (os primeiros pela facilidade de passar pelas malhas sem serem capturados e os últimos porque vão de encontro à rede, mas não ficam emalhados), alcançando seu valor máximo no comprimento médio de seleção (lc) (Fonteles-Filho, 1989).

As probabilidades de as redes de espera com os dois tamanhos de malha estudados capturarem indivíduos com comprimento de captura inferior ao mínimo foram calculadas considerando-se o comprimento mínimo de captura $(\operatorname{lmc})$ igual a $15 \mathrm{~cm}$, comprimento médio de seleção, e o desvio padrão da curva de seleção $(s)$ de 3,939 cm. Em ambos os casos, as probabilidades foram muito baixas e corresponderam a 1,32\% e 0,03\% para as malhas de $5 \mathrm{~cm}$ e de $6 \mathrm{~cm}$, respectivamente (Tabela III).

Tabela III - Probabilidades de capturar indivíduos com comprimento inferior ao mínimo, calculadas pela aplicação do teste $z$ das redes de espera com malhas de $5 \mathrm{~cm}$ e $6 \mathrm{~cm}$ entre nós opostos utilizadas na captura da sardinha-bandeira, Opisthonema oglinum (Lesueur, 1818), na Praia da Caponga, de agosto a outubro de 2014

\begin{tabular}{c|c|c|c|c}
\hline $\begin{array}{c}\text { Tamanho da } \\
\text { malha }(\mathrm{cm})\end{array}$ & $\begin{array}{c}\text { Comprimento } \\
\text { médio de seleção } \\
(l c)\end{array}$ & $\begin{array}{c}\text { Valores } \\
\text { de } z\end{array}$ & $\mathrm{~A}^{*}$ & $P(l<15 \mathrm{~cm})$ \\
\hline 5 & 23,73 & $-2,22$ & 0,4868 & $(0,5-0,4868) \times 100 \%=1,32 \%$ \\
6 & 28,47 & $-3,42$ & 0,4997 & $(0,5-0,4997) \times 100 \%=0,03 \%$ \\
\hline
\end{tabular}

Fonte: elaborada pelo autor.

*Valor tabelado da área de uma distribuição normal padrão ou reduzida.

O comprimento mínimo de captura $(\mathrm{lcm})$ de $15 \mathrm{~cm}$ foi usado considerando-se o que estabelece a Instrução Normativa no 53/05 do Ministério do Meio Ambiente (Brasil, 2005) para espécies marinhas e estuarinas do litoral do Sudeste e Sul do Brasil. Esse valor foi 
adotado devido à inexistência de legislação para peixes do Nordeste brasileiro. Entretanto, é importante destacar que, no trabalho de Mota-Alves e Sawaya (1975), a primeira maturação sexual da sardinha-bandeira do Ceará ocorre em $50 \%$ da população, na classe de comprimento total de $14,2 \mathrm{~cm}$ a 14,8 cm. Para Vazzoler (1981), o comprimento da primeira maturação sexual é definido como aquele em que 50\% dos indivíduos da população entram ativamente em processo de maturação sexual. Assim, as informações de Mota-Alves e Sawaya (14,2 a 14,8 cm) e da instrução normativa $(15 \mathrm{~cm})$ não são muito diferentes.

Fonteles-Filho (1989) afirma que, sendo o comprimento médio na primeira maturidade sexual o parâmetro que delimita as fases jovem e adulta na população, uma das principais aplicações do estudo da seletividade seria o ajustamento do aparelho de pesca, no sentido de que o comprimento médio de seleção seja o mais próximo possível do valor de primeira maturação sexual, para que o estoque capturável contenha uma proporção mínima de indivíduos imaturos. Logo, levando em consideração os resultados obtidos neste trabalho, $l c(23,7 \mathrm{~cm}$ e $28,5 \mathrm{~cm})>\operatorname{lmc}(15 \mathrm{~cm})$, pode-se verificar que tanto a malha de $5 \mathrm{~cm}$ como a de $6 \mathrm{~cm}$ capturam mais de $50 \%$ de indivíduos adultos, ou seja, essas malhas são indicadas para as redes empregadas na pesca da sardinha-bandeira.

\section{CONCLUSÕES}

As médias do comprimento total da sardinha-bandeira capturada na Praia da Caponga, com rede de espera de malha de $5 \mathrm{~cm}$ entre nós opostos, foram estatisticamente menores do que as dos indivíduos capturados com a malha de $6 \mathrm{~cm}$.

Comprova-se, com base nos comprimentos médios, na amplitude e nas equações das curvas de seleção das redes de espera, que o uso da malha de $6 \mathrm{~cm}$ tende a capturar indivíduos maiores. Ainda assim, tanto as redes de espera de malha de $5 \mathrm{~cm}$ quanto as de $6 \mathrm{~cm}$ apresentam probabilidades muito baixas de capturar indivíduos com comprimento inferior a $15 \mathrm{~cm}$, valor do comprimento mínimo de captura preconizado na literatura.

O estoque pesqueiro capturável da sardinha-bandeira na Praia da Caponga foi composto na sua totalidade por indivíduos adultos, com comprimento superior a $15 \mathrm{~cm}$.

Ainda existem poucas informações sobre a pesca e a biologia da sardinha-bandeira na região, havendo necessidade de mais estudos de Biologia Pesqueira/Dinâmica Populacional, como os de biologia reprodutiva, alimentação, mortalidade, crescimento e recrutamento. Com base nessas informações, será possível realizar a avaliação do estoque, a fim de gerar informações úteis ao gerenciamento da pesca desse recurso, buscando uma exploração sustentável. Essas informações se revestem de grande importância para a manutenção do estoque da sardinha-bandeira em equilíbrio sustentável, devendo ser levadas em consideração no estabelecimento de legislação específica.

\section{REFERÊNCIAS BIBLIOGRÁFICAS}

Bjordal, A. Uso de medidas técnicas em la pesca responsable: regulación de artes de pesca, in Cochrane, K.L. (ed.). Guia del Administrador Pesquero. Medidas de ordenacion y suaplicacion. FAO Documento Técnico de Pesca nº 424, Roma: FAO, 231 p., 2005.

Brasil. Instrução Normativa ${ }^{\circ}$ 53, de 22 de novembro de 2005. Estabelece o tamanho mínimo de captura de espécies marinhas e estuarinas do litoral Sudeste e Sul do Brasil. Diário Oficial [da] União, Ministério do Meio Ambiente (MMA), Brasília, p. 87, 24 nov. 2005. 
Brasil. Boletim da estatística da pesca marítima e estuarina do Nordeste do Brasil - 2006. Monitoramento da atividade pesqueira no estado do Ceará. p. 131-165. Instituto Brasileiro do Meio Ambiente e dos Recursos Naturais Renováveis (Ibama), 2006.

Brasil. Estatística da pesca 2006. Brasil: grandes regiões e unidades da federação. Pesca extrativa marinha. p. 4-34. Instituto Brasileiro do Meio Ambiente e dos Recursos Naturais Renováveis (Ibama), 2007.

Brasil. Ministério da Pesca e Aquicultura. Boletim Estatístico da Pesca e Aquicultura 2011. Brasília, 2013.

Camara, J.J.C.; Rodrigues, A.M.; Campos, E.C.; Santos, R.A.; Barbosa, J.M. \& MandelliJúnior, J. Pesca seletiva do tambiú, Astyanax bimaculatus Linnaeus, 1758 (Characiformes, Characidae), com a utilização de redes de emalhar, na represa de Ibitinga, rio Tietê, estado de São Paulo, Brasil. Bol. do Instituto de Pesca, São Paulo, v. 18 (único), p. 51-60, 1991.

Capistrano-Sobrinho, D.; Caetano-Pereira, A.C.S.; Prado, J.P.S.; Motta, A.L.V.; Boelter, J.F. \& Cavalheiro, J.M.O. Composição química e avaliação do processo de liofilização do filé de sardinha-laje (Opisthonema oglinum). Rev. de Biol. e Farm., João Pessoa, v. 5, n. 2, p. 50-58, 2011.

Castello, J.P. Gestão sustentável dos recursos pesqueiros, isto é possível? Pan-American Journal of Aquatic Sciences, Montevideo, v. 2, n. 1, p. 47-52, 2007.

Castro e Silva, M.M. Caracterização da pesca artesanal na costa do estado do Ceará, Brasil. 2004. Tese de doutorado, Programa de Pós-Graduação em Ecologia e Recursos Naturais, Universidade Federal de São Carlos, 262 p., São Carlos, 2004.

Ceará. Perfil básico municipal: Cascavel, in Instituto de Pesquisa e Estratégia Econômica do Ceará - Ipece. 2013. 17 p.

Figueiredo, J.L. \& Menezes, N.A. Manual de peixes marinhos do Sudeste do Brasil: II. Teleostei (1). Museu de Zoologia da Universidade de São Paulo, 110 p., São Paulo, 1978.

Fonteles-Filho, A.A. Oceanografia, biologia e dinâmica populacional de recursos pesqueiros. Fortaleza: Expressão Gráfica. 460 p., 2011.

Fonteles-Filho, A.A. Recursos pesqueiros: biologia e dinâmica populacional. Fortaleza: Imprensa Oficial do Ceará, 296 p., 1989.

Froese, R. \& Pauly, D. (eds.). FishBase. World Wide Web electronic publication. 2019. Disponível em: http://www.fishbase.org/summary/Opisthonema-oglinum.html. Version (12/2019). Acesso em: 13 fev. 2019.

Froese, R. \& Pauly, D. (eds.). FishBase. World Wide Web electronic publication. 2009. Disponível em: www.fishbase.org. Version (09/2009). Acesso em: 6 jan. 2015.

Furtado-Ogawa, E. Alimentação da sardinha-bandeira, Opisthonema oglinum (Lesueur), no estado do Ceará. Arq. de Ciên. do Mar, Fortaleza, v. 10, n. 2, p. 201-202, 1970.

Holt, S.J. A method for determining gear selectivity and its application, p. 106-115, In International Commission for the Northwest Atlantic Fisheries, 1957, Lisbon. Proceedings... Dartmouth: Rolph-Clark-Stone, Maritimes, Limited, 1963. Special Publication n ${ }^{\circ}$ 5. ICNAF/ ICES/FAO, v. 2, 228 p., 1963.

Instituto de Desenvolvimento Sustentável Mamirauá. BioEstat 5.3. Disponível em: http:// www.mamiraua.org.br/pt-br/downloads/programas/. Acesso em: 13 fev. 2019. 
Ihering, R.V. Aspectos da pesca no litoral nordestino. A Voz do Mar, Rio de Janeiro, v. XI, n. 110, p. 228-229, 1932.

Kobayashi, R.K. Características da pesca artesanal na Praia da Caponga, município de Cascavel, estado do Ceará. Fortaleza: UFC, Relatório Técnico, 31 p., 2000.

Lessa, R. \& Nóbrega, M.F. Guia de identificação de peixes marinhos da região Nordeste. Recife: UFRPE-DIMAR, 128 p., 2000.

Lima, S.A.O. \& Andrade, H.A. Gillnet selectivity for forage fish with emphasis on manjuba (Opisthonema oglinum) in an estuary in the northeast of Brazil. Bol. do Instituto de Pesca, São Paulo, v. 44, n. 3, p. 1-12, 2018.

Lino, M.A.S. Estudo biológico-pesqueiro da manjuba, Opisthonema oglinum (Lesueur, 1818), da região de Itapissuma, Pernambuco. Dissertação de mestrado, Programa de Pós-Graduação em Recursos Pesqueiros e Aquicultura, Universidade Federal Rural de Pernambuco, 34 p., Recife, 2003.

Martins, J.C.; Juras, A.A.; Araújo, M.A.S.; Melo-Filho, A.S. \& Cintra, I.H.A. Seletividade da rede malhadeira-fixa para a captura do mapará, Hypophthalmus marginatus, no reservatório da usina hidrelétrica de Tucuruí, estado do Pará, Brasil. Bol. do Instituto de Pesca, São Paulo, v. 37, n. 2, p. 123-133, 2011.

Menezes, M.F. Aspectos da pesca artesanal de algumas espécies marinhas no estado do Ceará. Bol. da Estação de Biologia Marinha da Universidade Federal do Ceará, Fortaleza, v. 17, p. 1-11, 1968.

Mota-Alves, M.I. \& Sawaya, P. Aspectos do aparelho digestivo e da alimentação de Opisthonema oglinum (Lesueur) (Pisces Clupeidae). Arq. de Ciên. do Mar, Fortaleza, v. 14, n. 2, p. 135-144, 1974.

Mota-Alves, M.I. \& Sawaya, P. Sobre a reprodução da sardinha-bandeira, Opisthonema oglinum (Lesueur), na costa do estado do Ceará (Brasil). Arq. de Ciên. do Mar, Fortaleza, v. 15, n. 1, p. 19-28, 1975.

Puzzi, A. \& Silva, M.R.G. Seletividade em redes de emalhar e dimensionamento do tamanho de malha para a captura da corvina Micropogonias furnieri (Desmarest, 1823). Bol. do Instituto de Pesca, São Paulo, v. 8 (único), p.139-156, 1981.

Sá, G.G. Primeira abordagem sobre a pesca da sardinha-bandeira, Opisthonema oglinum (Lesueur, 1818), em Caponga, Cascavel-CE. 2010. Monografia de graduação, curso de graduação em Engenharia de Pesca, Centro de Ciências Agrárias, Universidade Federal do Ceará, 59 p., Fortaleza, 2010.

Teixeira, S.R.A. Estudo biológico-pesqueiro da sardinha-bandeira, Opisthonema oglinum (Lesueur, 1818), da região de Cascavel, Ceará, Brasil. 2013. Monografia de graduação, curso de graduação em Engenharia de Pesca, Centro de Ciências Agrárias, Universidade Federal do Ceará, 30 p., Fortaleza, 2013.

Vazzoler, A.E.A.M. Manual de métodos para estudos biológicos de populações de peixes: reprodução e crescimento. Brasília: CNPq, Programa Nacional de Zoologia, 108 p., 1981.

Vieira, A.C.; Litivak, A.C.; Lucena, F.P. \& Oliveira, V.S. Produção de sardinha-laje (Opisthomena oglinum) no Nordeste do Brasil, in Anais da X Jornada de Ensino, Pesquisa e Extensão, Recife, 2010. 
Whitehead, P.J.P. Clupeoid fishes of the world (suborder Clupeioidei). An ananotated and illustrated catalogue of the herrings, sardines, pilchards, sprats, shads, anchovies and wolf-herrings, in FAO species catalogue, v. 7, FAO Fisheries Synopsis, 303 p., Roma, 1985. 